\title{
System for Measuring Finger Force Profiles for Dexterity Assessment
}

\author{
Milica M. Badža, Marija M. Novičić, Milica Đurić-Jovičić, \\ Milica M. Janković, Member, IEEE, and Mirjana B. Popović
}

\begin{abstract}
We propose a novel system for measuring finger force profiles for dexterity assessment. The system consists of a software application and encased hardware. The sensing part of the system consists of ten high sensitivity strain gage force sensors, one for each finger. The developed application controls turning ON/OFF of the LEDs (each LED corresponds to one finger) to signalize the user which finger should press the corresponding strain gage force sensor. Linearity, repeatability, and sensitivity to position pressure of the strain gage force sensors were tested to prove the system reliability. The potential usage of the system with the assessment of quantitative parameters was demonstrated on two healthy subjects.
\end{abstract}

Keywords - finger dexterity, finger force, strain gage force sensor, quantitative assessment.

\section{INTRODUCTION}

$\mathrm{T}$ HE hand is one the most complex and amazing examples of natural engineering in the human body: providing a powerful grip, yet allowing us to manipulate small objects with great precision and speed. However, hand movements, particularly fingers, are also frequently affected after a stroke or neurodegenerative diseases, as well as different types of trauma and injuries [1]. In diseases like rheumatoid arthritis, the hands are frequently affected by pain, a feeling of weakness and mobility difficulties [2].

Recovery or progress of the disease is typically slow and invisible to the human eye. Furthermore, if it was done by

Paper received May 15, 2019; revised August 12, 2019; accepted August 16, 2019. Date of publication December 30, 2019. The associate editor coordinating the review of this manuscript and approving it for publication was Prof. Miroslav Lutovac.

This paper is a revised and expanded version of the paper presented at the 26th Telecommunications Forum TELFOR 2018 [20].

This project is financially supported by the Ministry of Education, Science and Technological Development of the Republic of Serbia (no. OS 175016).

Milica M. Badža (corresponding author), is with the School of Electrical Engineering, University of Belgrade, Bul. kralja Aleksandra 73, 11120 Belgrade, Serbia (e-mail: milica.badza@yahoo.com).

Marija M. Novičić (corresponding author), University of Belgrade - School of Electrical Engineering, Bulevar kralja Aleksandra 73, 11120 Belgrade, Serbia (e-mail: marijanovicic5@gmail.com).

Milica Djurić-Jovičić is with the Innovation Center, University of Belgrade - School of Electrical Engineering, Serbia (e-mail: milica.djuric@etf.rs).

Milica M. Janković, University of Belgrade - School of Electrical Engineering, Bulevar kralja Aleksandra 73, 11120 Belgrade, Serbia (e-mail: piperski@etf.rs).

Mirjana B. Popović, University of Belgrade - School of Electrical Engineering, Bulevar kralja Aleksandra 73, 11120 Belgrade, Serbia (e-mail: mpo@etf.rs). visual observation, the assessment is susceptible to human subjectivity. Having a sensor system which could be used for objective assessment of our motor performance and potential impairments can help clinicians to track small and slow changes in the recovery or response to therapy, quantify surgery effects or physical therapy after hand trauma or disease [3].

There are different devices described in the literature designed for measuring finger flexion force [4-6], measuring both single finger and whole hand extension force [7], devices directly attached to the finger or as a glove [8-10]. Aside from studies concerning voluntary finger forces, involuntary "crosstalk" between fingers was investigated for measuring synergistic finger contractions [11]. Besides force, studies were also analyzing finger speed and regularity of finger movements, most frequently during different forms of finger tapping tasks, such as during simulating piano playing [12]. Such movements were studied in patients with Parkinson's disease and a control group of healthy subjects, by using motion capture optical system and markers attached to anatomical landmark points. Another group developed a system for estimating piano-playing-like motions, designed in the form of four electronic touch plates in a fan shape and a hand rest as the fifth plate [13]. A haptic glove paired with designed virtual reality games based on piano-like movements was also used to train poststroke patients and assess dexterity [14]. Having force sensors integrated into a haptic glove is useful not only for force assessment but also as tactile feedback connecting a tactile sensation detected on fingertips with the virtual world in the therapy game [1517]. A similar setup was used to restore a lost tactile sensation in the fingers to improve grasp force control of the patients with Multiple Sclerosis [18]. Grasp force can also be improved using electrical stimulation [19].

However, such wearable technologies can be challenging for clinical use when it comes to system setup, sensor mounting, maintenance and scaling to all sizes. To become integrated into a clinical assessment routine, a system should be simple to use, requiring minimal setup time and skills, and possibly function on a plug-and-play principle. Despite the progress of the technology and decrease in prices for the different sensors, currently, there is no commercially available or standardized system for dexterity assessment based on finger forces to fulfill such demands.

In this paper, we propose a novel system for measuring finger force profiles for dexterity assessment. The system includes load cell force sensors built in the hardware casing suitable for assessment of piano-like finger movements. 
The software is developed to support the design of the experimental tasks as well as signal acquisition. This paper represents an expanded version of the paper presented at the 26th Telecommunications Forum TELFOR 2018 [20]. The system was tested on two healthy subjects (for both hands), testing its performances, repetitiveness, and reliability.

\section{METHOD}

\section{A. System architecture}

The system consists of the software application and encased hardware, which includes ten force sensors, ten LEDs, amplifier and A/D card. The block diagram of the system is presented in Fig. 1. Only the right-hand part of the system is shown, while the left-hand part is symmetrical.

The developed application controls turning ON/OFF of the LEDs (each LED corresponds to one finger) to signalize the user which finger should press the corresponding strain gage force sensor.

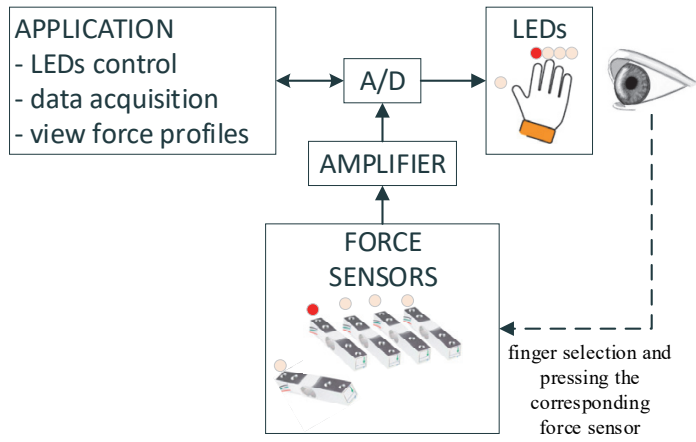

Fig. 1. The block diagram for the right-hand part of the system.

The sensing part of the system consists of the high sensitivity strain gage force sensors (Load Cell AMI5, Keli Sensing Technology, China) with a maximum force of 50 $\mathrm{N}$, and sensitivity $2 \pm 0.2 \mathrm{mV} / \mathrm{V}$. The force sensor is selected according to the fact that the maximal pinch force is less than $50 \mathrm{~N}$ [21]. The voltage outputs of the sensors are amplified because their values are in range from 0 to $25 \mathrm{mV}$. The differential gain of INA 114 amplifier (Texas Instruments, USA) is equal to 500. Output signals are further discretized using NI USB 6009 (National Instruments, Texas, USA). Afterward, they are processed and stored as force profiles by the developed application. The whole hardware is stored in a dedicated, precisely designed PVC casing. Dimensions of the casing are $48 \times 37 \times 5 \mathrm{~cm}$. The positions of the sensors in the casing are suited to the anatomy of the right and left hand separately. A soft, raised pad for the examinee's palm is placed in front of the sensors.

\section{B. Software description}

The user-friendly application is developed in LabVIEW ver. 2017 environment (National Instruments Inc., Austin, USA). The flowchart of the user interface is presented in Fig. 2.

At the beginning of the program execution, the user is asked to choose between two options: Sensor calibration and Testing. In the Sensor calibration option, force sensor's linearity is examined, and recalibration could be performed if needed. Sensor calibration option returns new values for the parameters of the linear fit, slope and intercept.

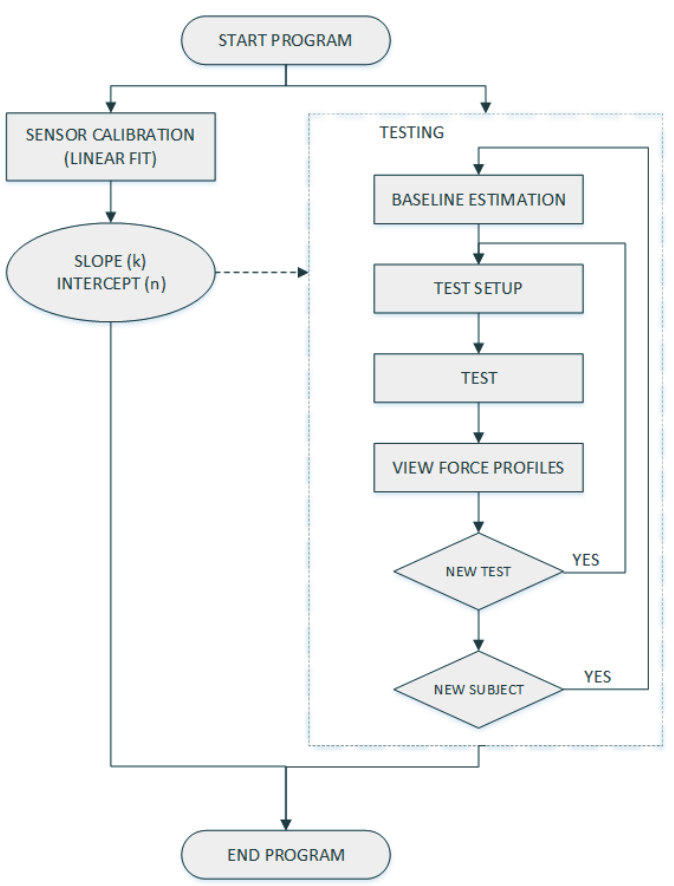

Fig. 2. The flow chart of the user interface.

The first step in the Testing option is baseline estimation and it is a necessary step for starting a new subject study. Baseline estimation represents the measured force without the load (no contact between the fingers and the load cells). It is estimated as the mean value of the force in the time interval of $1 \mathrm{~s}$, with the sample rate of $100 \mathrm{~Hz}$. This force mean value is used for the baseline correction of the force in the "test setup" step.

At the beginning of the ,test setup" step, the following variables can be selected: 1) a file path for data logging, 2) selection of the hand (left or right), 3) number of trials $N$ for turning ON/OFF of the LED, 4) the duration of ,LED turned ON" phase and 5) test type: the maximum force test or the dexterity test.

Before the "dexterity testing", the "maximum force test" should be performed for each finger in order to be used for force normalization.

The "dexterity test" procedure includes recording of the force profile for each finger. During the test, the user is asked to press the appropriate load cell $N$ times with each finger of the selected hand. Application randomly turns each LED ON or OFF $N$ times in total (not necessarily consecutively). Fingers' force profiles are acquired by a sample rate of $100 \mathrm{~Hz}$, corrected relating to the ,baseline force", normalized, and saved in the spreadsheet file (including the finger labels).

At the end of the "test" step, it is possible to view force profiles and calculated force parameters for each finger (for all trials). An example of a force profile result for index finger is shown in Fig. 3.

For each trial, the following characteristic parameters are automatically calculated in the designed application: time reaction $(T R)$, peak of force $(P$, expressed as a percentage of maximal finger force), rise time ( $R T$, time interval in which the force increases from $10 \%$ to $90 \%$ of peak), fall 
time ( $F T$, time interval in which the force decreases from $90 \%$ to $10 \%$ of peak), duration of the response $(D)$ and full width of half maximum (FWHM).

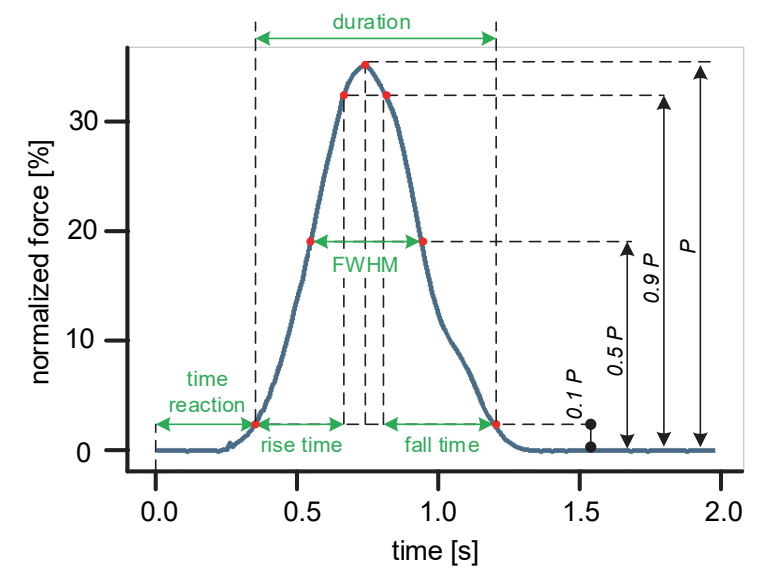

Fig. 3. An example of a force profile for index finger and characteristic parameters.

\section{Experiment description and statistics}

The potential usage of the system is illustrated through an experiment example. The experiment was performed on two healthy subjects, one female and one male subject, both 22 years old, and both right-hand writers. The written informed consent form was signed. The experiment is carried out in accordance with the ethical standards of the Declaration of Helsinki.

The examinee was sitting straight in a chair in front of a desk, as shown in Fig. 4. The palm is leaning on the pad, and fingers are positioned directly above the sensors, Fig. 5. In this way, the influence of other hand movements is eliminated, and only the finger movements are observed and analyzed.

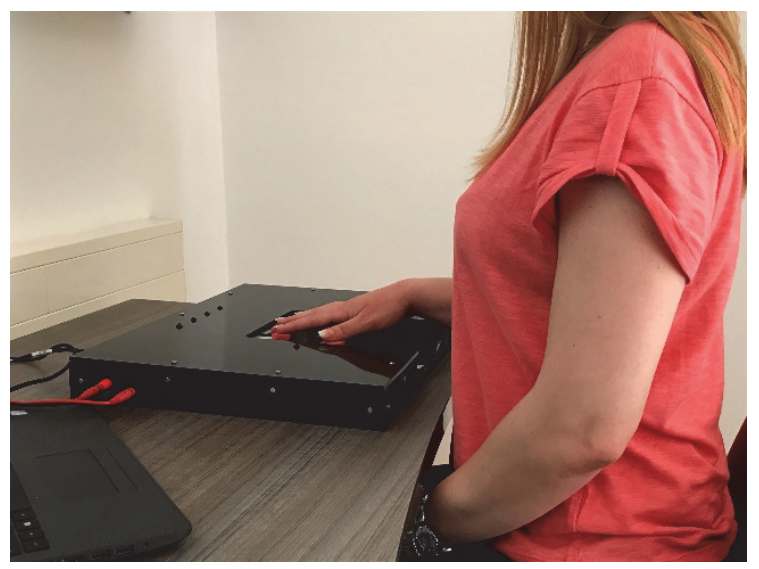

Fig. 4. The position of the examinee during the testing.

The first measurement that the subject performs is the "maximum force test" for each finger. The maximum force is used in statistical analysis (Section IIIC). Afterward, ten test sessions for the "dexterity test": were performed. One session corresponds to one recording, and each session had 20 trials for each finger in a random order. The "dexterity test" is repeated for both hands independently. The duration of „LED turned ON" phase was set to 2 s. The pause between tests was 5 minutes to avoid fatigue impact on the measurement.

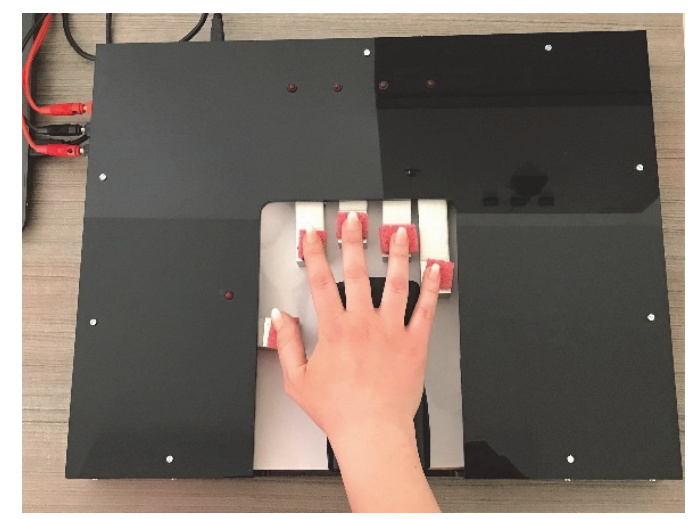

Fig. 5. The position of the hand during the examination.

Statistical analysis was performed using the software environment $\mathrm{R}$ ver. 3.5.1, Foundation for Statistical Computing. The mean value and the standard deviation were calculated for the previously listed response parameters. The force profiles of all trials were aligned to the peak, averaged and shifted to the mean value of the time reaction in order to better visualize the obtained data.

\section{RESULTS}

\section{A. Linearity, repeatability, and sensitivity of strain gage force sensor to the pressure point}

To test linearity and repeatability of applied strain gage force sensors, we have measured their voltage output for 10 different loads (in range of $0.05 \mathrm{~N}$ to $50 \mathrm{~N}$ ), going from the lowest to the highest, and back. This procedure was repeated 10 times and averaged results (mean \pm standard deviation) are presented in Fig. 6. The curve shows the result of linear fitting $(y=\mathrm{k} x+\mathrm{n}, \mathrm{k}=0.21 V / N, \mathrm{n}=0.2982 V$, the coefficient of determination $\mathrm{R}^{2}=0.99$ ).

The sensitivity of the strain gage force sensor to the pressure point position was tested, Fig. 7 . The sensitivity is less than $2 \%$, even for very low loads $(0.05 \mathrm{~N})$.

We have measured the voltage output of sensors for 10 different loads (in the range $0.05 \mathrm{~N}$ to $25 \mathrm{~N}$ ) on 9 different positions on the sensor, and the force was measured. The upper limit for force testing was $25 \mathrm{~N}$ because the physical dimensions of the appropriate weight were comparable to the pressure area of the sensor.

During all test procedures, force information was acquired using the aforementioned Sensor calibration option of the developed application described in Section II B.

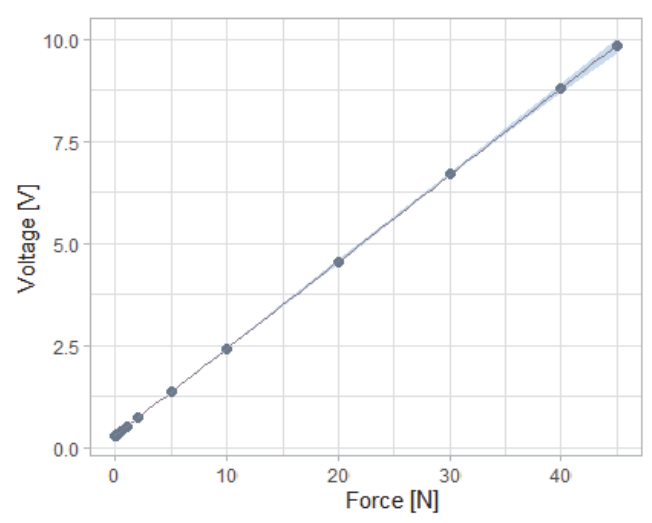

Fig. 6. Results of strain gage force sensor linearity. 


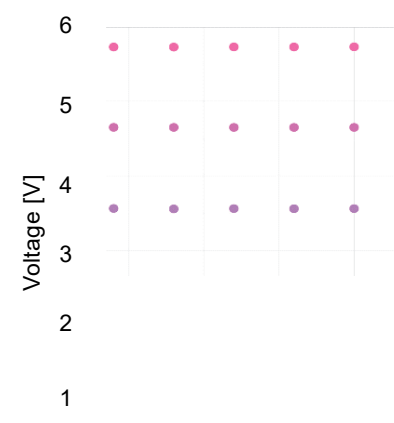

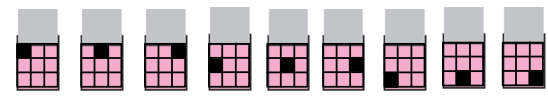

Position of the pressure point

Fig. 7. The sensitivity of the strain gage force sensor to the pressure point. Finger position on the sensor is presented along the $x$-axis.

\section{B. User interface}

The user interface at the beginning of Testing option is shown in Fig. 8A). After choosing the "test setup" option, a window shown in Fig. 8B) pops up. After initial settings, the user selects "dexterity test" or "maximum force test". A screenshot of the user interface in the "dexterity test" step is presented in Fig. 8C). The indicator of the user interface is turned $\mathrm{ON}$ at the same time as the corresponding LED. On the graph below drawn hands with light indicators, it is possible to track force responses in real time.

After the test is finished, the user is provided with the option of generating the test results. In the "results" step, it is possible to view force profiles for each finger on a separate graph, Fig. 9. Each graph shows the force profiles for all of the $N$ trials. Finally, Fig. 10 shows tables with the calculated response parameters mentioned previously in the Section II B.

\section{Statistical results}

Descriptive statistics (mean value \pm standard deviation) of force parameters are presented in Table 1 (right hand), and in Table 2 (left hand) for both examinees.

Fig. 11A) shows aligned to the peak, averaged and shifted to the mean time reaction force profiles for all fingers in all trials, for right hand and both examinees. The aligned and averaged force profiles for left hand are shown in Fig. 11B). The relative error of standard deviations for calculated parameters, except for peak of force, from Table 1 and Table 2 is in the range 10-23\%. It could be observed that parameters $D$, and $F W H M$ show less variability than others $(10-15 \%)$. The observed relative error of standard deviation for parameter $P$ for comfortable single finger force is consistent with results found in the paper where strain gauge force sensors were employed for measuring flexion and extension force with fingers firmly attached to the sensors [11]. To our knowledge, there is no system described in literature that accentuates measurements and statistical analysis of finger force profiles based on parameters described in this paper. Thus, our system takes a novel approach in dexterity assessment.

\section{CONCLUSION}

A system for objective assessment of finger forces is presented. The linearity and repeatability of used load cells have been tested. The linear response does not depend on the pressure point of the weight. The shape of the finger profile is similar for all fingers, Fig. 11, but inter-subject and intra-subject variability should be more investigated in the future.

Further research will include an examination on more healthy subjects (in different motor, cognitive or environmental conditions, left $v s$ right hand comparison), and patients with different neurological diseases. In order to gain a further insight into the condition of those patients, the system will be adapted to estimate synergy between fingers thus measuring their "crosstalk".
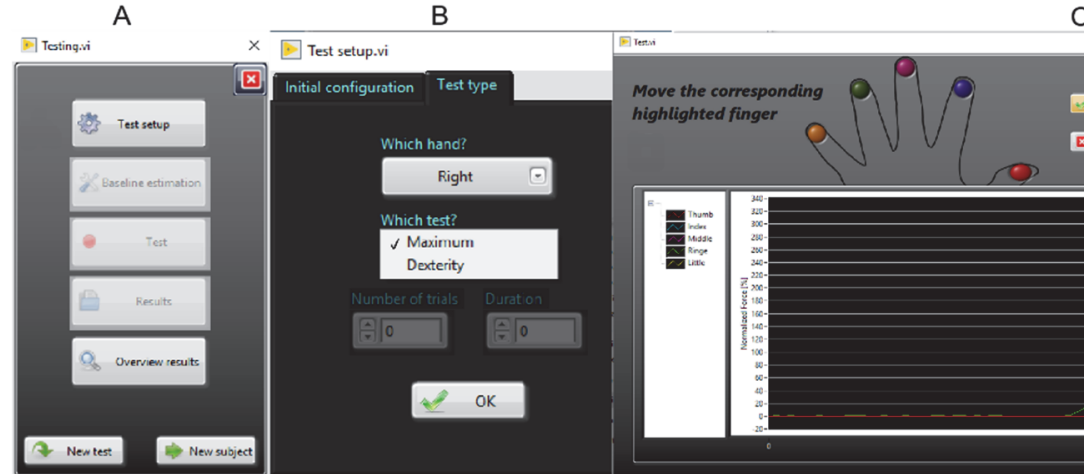

C

Fig. 8. A) The user interface at the beginning of the Testing option; B) Window that pops up after choosing the "test setup" step; C) User interface in the "dexterity test" step - ring finger of right hand is selected

("green" indicator is ON on the user interface). 


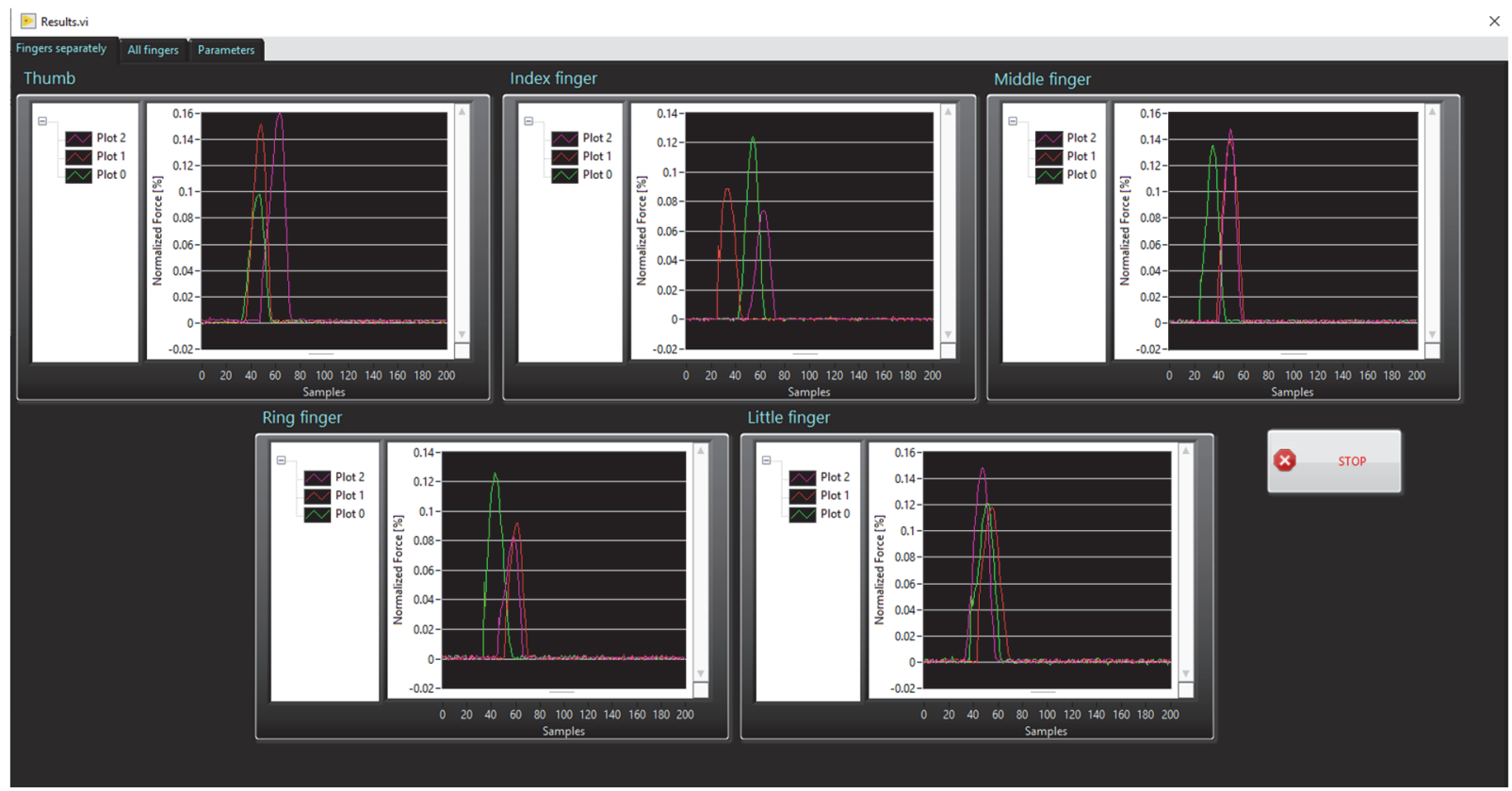

Fig. 9. Graphs for each finger separately with 3 trials in "results" step window.

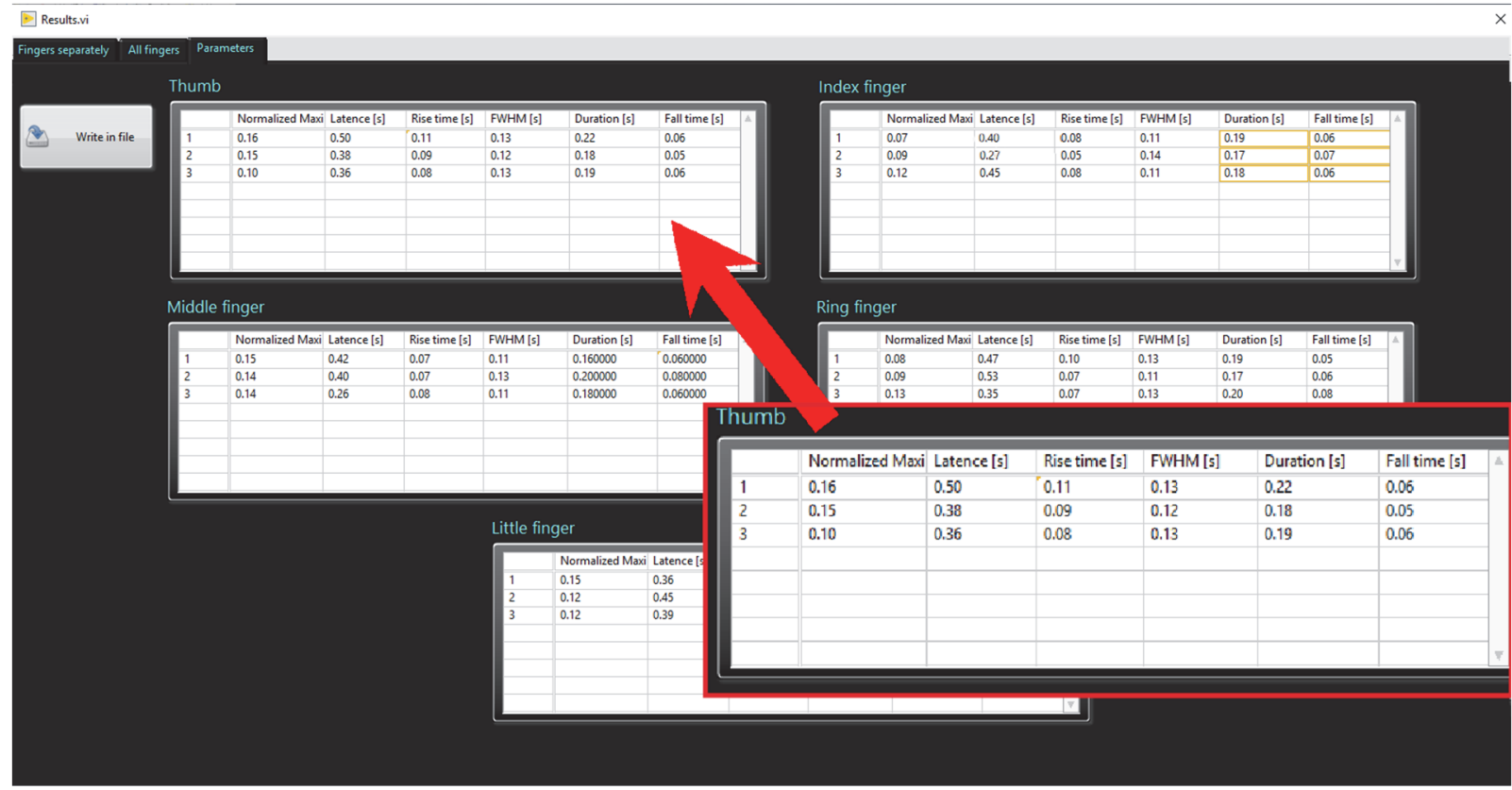

Fig. 10. Table with calculated parameters for each finger separately with 3 trials in "results" step window.

TABLE 1: MEAN VALUES \pm STANDARD DEVIATION OF FORCE PARAMETERS FOR THE FIRST/SECOND SUBJECT FOR RIGHT HAND.

\begin{tabular}{l|l|l|l|l|l}
\hline Parameters & Thumb & Index & Middle & Ring \\
\hline$R T[\mathrm{~ms}]$ & $142 \pm 23 / 82 \pm 19$ & $133 \pm 19 / 70 \pm 17$ & $131 \pm 23 / 64 \pm 15$ & $132 \pm 34 / 74 \pm 17$ & $139 \pm 27 / 74 \pm 16$ \\
$F T[\mathrm{~ms}]$ & $80 \pm 14 / 58 \pm 11$ & $78 \pm 9 / 50 \pm 9$ & $77 \pm 15 / 56 \pm 9$ & $84 \pm 15 / 60 \pm 12$ & $92 \pm 17 / 64 \pm 12$ \\
$P[\%]$ & $14 \pm 6 / 19 \pm 6$ & $15 \pm 5 / 13 \pm 5$ & $18 \pm 5 / 13 \pm 5$ & $15 \pm 4 / 19 \pm 6$ & $18 \pm 7 / 24 \pm 8$ \\
$D[\mathrm{~ms}]$ & $286 \pm 35 / 180 \pm 27$ & $274 \pm 29 / 154 \pm 23$ & $270 \pm 34 / 156 \pm 22$ & $283 \pm 41 / 176 \pm 23$ & $302 \pm 39 / 179 \pm 23$ \\
$T R[\mathrm{~ms}]$ & $437 \pm 78 / 559 \pm 122$ & $429 \pm 75 / 460 \pm 101$ & $428 \pm 87 / 496 \pm 110$ & $397 \pm 97 / 482 \pm 108$ & $437 \pm 80 / 441 \pm 64$ \\
$F W H M[\mathrm{~ms}]$ & $165 \pm 24 / 109 \pm 16$ & $162 \pm 17 / 93 \pm 14$ & $156 \pm 21 / 98 \pm 17$ & $179 \pm 29 / 108 \pm 16$ & $187 \pm 27 / 109 \pm 17$ \\
\hline
\end{tabular}

TABLE 2: MEAN VALUES \pm STANDARD DEVIATION OF FORCE PARAMETERS FOR THE FIRST/SECOND SUBJECT FOR LEFT HAND.

\begin{tabular}{|l|l|l|l|l|l}
\hline Parameters & Thumb & Index & Middle & Ring & Little \\
\hline$R T[\mathrm{~ms}]$ & $151 \pm 31 / 91 \pm 21$ & $135 \pm 43 / 72 \pm 17$ & $140 \pm 24 / 72 \pm 16$ & $149 \pm 34 / 71 \pm 16$ & $150 \pm 26 / 80 \pm 18$ \\
$F T[\mathrm{~ms}]$ & $85 \pm 18 / 63 \pm 10$ & $79 \pm 15 / 65 \pm 14$ & $140 \pm 24 / 63 \pm 9$ & $83 \pm 14 / 65 \pm 10$ & $85 \pm 15 / 62 \pm 11$ \\
$P[\%]$ & $20 \pm 7 / 19 \pm 4$ & $9 \pm 2 / 13 \pm 3$ & $15 \pm 5 / 13 \pm 3$ & $15 \pm 4 / 17 \pm 4$ & $17 \pm 8 / 17 \pm 4$ \\
$D[\mathrm{~ms}]$ & $312 \pm 46 / 204 \pm 28$ & $278 \pm 41 / 179 \pm 32$ & $289 \pm 32 / 173 \pm 27$ & $300 \pm 41 / 177 \pm 26$ & $307 \pm 39 / 190 \pm 27$ \\
$T R[\mathrm{~ms}]$ & $507 \pm 94 / 520 \pm 92$ & $407 \pm 97 / 443 \pm 101$ & $439 \pm 103 / 428 \pm 93$ & $397 \pm 100 / 435 \pm 96$ & $457 \pm 94 / 432 \pm 95$ \\
$F W H M[\mathrm{~ms}]$ & $187 \pm 27 / 129 \pm 19$ & $165 \pm 18 / 111 \pm 19$ & $172 \pm 21 / 106 \pm 19$ & $178 \pm 25 / 112 \pm 19$ & $185 \pm 26 / 118 \pm 18$ \\
\hline
\end{tabular}



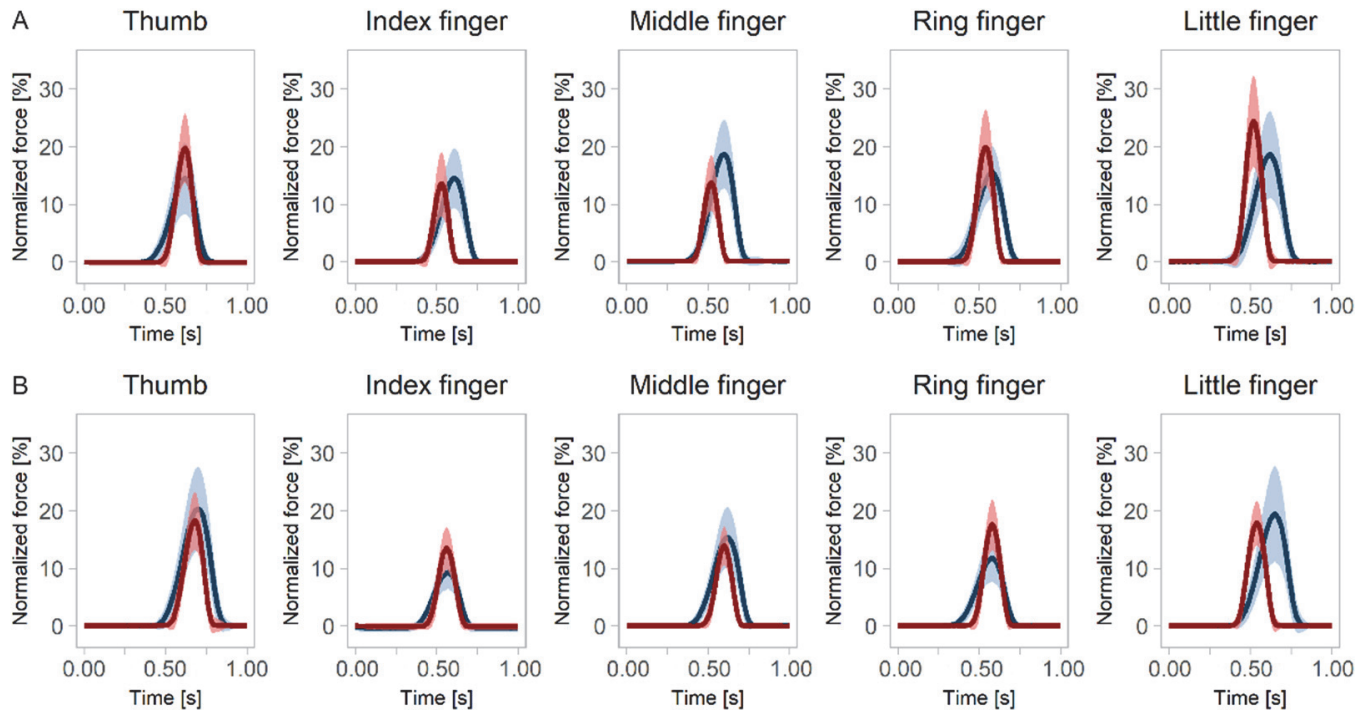

Fig. 11. Averaged and aligned force profiles for A) right and B) left hand for the first (blue) and second (red) subject.

\section{ACKNOWLEDGMENT}

We thank Novica Janković, researcher at the Faculty of Mechanical Engineering, University of Belgrade and Nikola Mališić, MSc in architectural engineering and Zoran Djordjević, technician and Vladislava Bobić researcher at the Innovation Center School of Electrical Engineering, University of Belgrade, for valuable comments and technical support.

\section{REFERENCES}

[1] R. Agostino, A. Currà, M. Giovannelli, N. Modugno, M. Manfredi, and A. Berardelli, "Impairment of individual finger movements in Parkinson's disease," Movement disorders, vol. 18, no. 5, pp. 560$565,2003$.

[2] E. Carmeli, H. Patish, and R. Coleman, "The aging hand," The Journals of Gerontology Series A: Biological Sciences and Medical Sciences, vol. 58, no. 2, pp. M146-M152, 2003.

[3] M. Đurić-Jovičić, N. Jovičić, S. Radovanović, M. Ječmenica-Lukić, M. Belić, M. Popović, and V.S. Kostić, "Finger and foot tapping sensor system for objective motor assessment," Vojnosanitetski pregled, vol. 75, no. 1, pp.68-77, 2018.

[4] U.M. Nordenskiol, G. Grimby, "Grip force in patients with rheumatoid arthritis and fibromyalgia and in healthy subjects. A study with the Grippit instrument," Scand J Rheumatol. 22:14-9, 1993.

[5] S. Kilbreath, R. Gorman, J. Raymond and S. Gandevia, "Distribution of the forces produced by motor unit activity in the human flexor digitorum profundus," J. Physiol. 543 289-96, 2002.

[6] S. Radhakrishnan and M. Nagaravindra, "Analysis of hand forces in health and disease during maximum isometric grasping of cylinders," Med. Biol. Eng. Comput. 31 372-6, 1993.

[7] S. Olandersson, H. Lundqvist, M. Bengtsson, M. Lundahl, A.J Baerveldt, and M. Hilliges, M., "Finger-force measurement-device for hand rehabilitation," Rehabilitation Robotics, ICORR 2005. 9th International Conference on, pp. 135-138, IEEE, 2005.

[8] K.L. Kilgore, R.T. Lauer, P. Hunter, "A Transducer for the Measurement of Finger Joint Moments," IEEE Transactions on Rehabilitation Engineering, vol. 6, no 4, pp. 424-429, 1998.

[9] S. N. P. de Silva, R. Mattar Jr, R. Bolliger Neto and C. A. M. Pereira, "Measurement of the flexing force of the fingers by a dynamic splint with a dynamometer," Clinics, vol. 60 381-8, 2005.
[10] J.D. da Silvia, A.A. de Carvalho, D.D. da Silva, "A Strain Gauge Tactile Sensor for Finger-Mounted Applications," IEEE Transaction on instrumentation and measurement, vol. 51, no. 1, pp. 18-22, 2002.

[11] R. Koiva, B. Hilsenbeck and C. Castellini, "FFLS: An accurate linear device for measuring synergistic finger contractions", In 2012 Annual International Conference of the IEEE Engineering in Medicine and Biology Society, pp. 531-534. IEEE, 2012.

[12] A. Jobbágya, P. Harcos, R. Karolya, G. Fazekas, “Analysis of fingertapping movement," Journal of Neuroscience Methods, vol. 141, pp. 29-39, 2005.

[13] S.R. Muir, R.D. Jones, J.H. Andreae, I.M. Donaldson, "Measurement and Analysis of Single and Multiple Finger Tapping in Normal and Parkinsonian Subjects," Parkinsonism Related Dis, vol. 1, pp. 89-96, 1995.

[14] C.Y. Lin, C.M. Tsai, P.C. Shih, H.C. Wu, "Development of a novel haptic glove for improving finger dexterity in poststroke rehabilitation," Technology and Health Care, vol. 1, no. 24, pp. 97. 103, 2016.

[15] S.A. Mascaro and H. Asada, "Finger Posture and Shear Force Measurement Using Fingernail Sensors: Initial Experimentation," International Conference on Robotics and Automation, vol. 2, pp 1857-1862, 2001.

[16] D. Prattichizzo, F. Chinello, C. Pacchierotti, and K. Minamizawa, "RemoTouch: A system for remote touch experience," IEEE International Symposium on Robot and Human Interactive Communication, pp. 676-679, 2010.

[17] F. Chinello, M. Malvezzi, C. Pacchierotti, and D. Prattichizzo, "A three DoFs wearable tactile display for exploration and manipulation of virtual objects," In Symposium on Haptic Interfaces for Virtual Environment and Teleoperator Systems, pp. 71-76, 2012.

[18] L. Jiang, M.R. Cutkosky, J. Ruutiainen, R. Raisamo, "Using haptic feedback to improve grasp force control in multiple sclerosis patients," IEEE transactions on Robotics, vol. 25, no. 3, pp. 593-601, 2009.

[19] A. J. Westerveld, A. C. Schouten, P.H. Veltink and H. van der Kooij, "Selectivity and resolution of surface electrical stimulation for grasp and release," IEEE Trans. Neural Syst. Rehabil. Eng., vol. 20, pp. 94-101, 2012.

[20] M. M. Badža, M.M. Novičić, M. Đurić-Jovičić, M. M. Janković and M. B. Popović, "System for Measuring Finger Force Profiles for Dexterity Assessment," In 2018 26th Telecommunications Forum (TELFOR), pp. 1-4. IEEE, 2018.

[21] T. Aoki, Y. Fukuoka, "Finger tapping ability in healthy elderly and young adults," Medicine and science in sports and exercise, vol. 42, no. 3, pp. 449-455, 2010. 